\title{
The iPad as an alternative to conventional technology for effective teaching
}

\author{
Ramanathan Sri Ranjan, Ph.D., P.Eng. \\ Professor, Department of Biosystems Engineering, University of Manitoba \\ Sri_ranjan@umanitoba.ca
}

\begin{abstract}
The iPad connected to a data projector in the class room can effectively replace conventional technology such as computers, overhead projectors, document cameras, and white boards. A selection of apps that can mimic the tasks of the existing technology will be demonstrated. The Keynote app can be used as the main tool for presenting the course content. The NotesPlus app can be used to replace the conventional overhead projector and the whiteboard. For those who are accustomed to the polythene rolls in the overhead projector, PaperView app will mimic the polythene roll with the ability to carry it around. A few apps to annotate .pdf files will be demonstrated. The BoardCam app will be used to mimic the document camera in teaching. The focus will be on demonstrating how the different apps are used seamlessly to present the course content to the students.
\end{abstract}

Keywords: Keynote app, Notes Plus app, Board Cam app, PaperView app

\section{INTRODUCTION}

Desktop PCs connected to data projectors have been used for over two decades as the primary delivery mechanism of course content in the classroom. However, they are limited in their ability to present the course content effectively. The use of technology to enhance classroom delivery of course material has come a long way with the advent of new technologies such as Tablet PCs and iPads. While each device has its advantages, the main features that will lead to mass adoption of the technology will be the ease of use and the multitude of tasks that each can do.

Traditionally, chalk boards and white boards were used as the primary method of showing complex derivations in class. With the back turned to the audience, it was not ideal as a teaching aid. Although the overhead projectors allowed one to face the audience as the teaching progressed, the mechanics of writing on a brightly lit surface was a strain on the eyes. When Tablet
PCs became available, they served the dual purpose of presenting the slides and serving as whiteboards. Many of the Tablet PCs had programmable buttons on the front panel which could be programmed to launch a sketching program such as Windows Paint. Using the programmable button, one could switch between the slideshow and the Paint program. Yet, the Tablet PCs were slow in their response and took a few minutes to boot up at the beginning of the class. On many occasions, the computer output was not readily recognized by the data projector. This destroyed the classroom ambiance.

Although, PowerPoint slides were used to present the main content of the course material, the derivations of complex equations and concepts had to be done live in the classroom. Sometimes, a diagram or a graph needed to be projected onto the screen using a document camera which was not readily available in all classrooms.

When the iPad 2 was released it had a front and rear facing camera. The multitasking capability with fast switching between the apps (applications) made it an ideal tool for teaching. When connected to a data projector, the iPad could easily function as a whiteboard, overhead projector, document camera and a computer to present the PowerPoint slides. The data projectors easily recognized the iPad output through a VGA adaptor. As soon as the cover is opened the iPad was ready for use. There was hardly any start-up time wasted before the lecture.

Although there are thousands of apps available to do different tasks, the ones presented in this paper were initially selected based on user reviews. This paper will describe the main features of the apps used in the course delivery. The PowerPoint slides were made into four slides/page printed handouts that were made available ahead of the lecture. The material presented in the slides were in point form and the detailed derivations and development of concepts were always done live in the classroom. The students were expected to add their own notes to these handouts. The students were surveyed on their experience with the use of the iPad as technological aid for teaching. Their responses were extremely positive with no negative response. 


\section{THE APPS USED IN THE CLASSROOM}

\subsection{Presenting the slides}

PowerPoint and “Keynote”, Apples counter part for PowerPoint, can both be used to present the slides. The "Documents to Go" app has the ability to open and edit Microsoft Office documents such as Word, Excel, and PowerPoint. Although, the Documents to Go app can present the PowerPoint slideshow on the iPad the transitions between the slides were not smooth compared to the Keynote app. The Keynote app easily opened the PowerPoint slides with minor font substitutions. The slide transitions in Keynote app were much smoother and touching a finger on the iPad screen activated a virtual laser pointer on the screen which was useful to highlight any points. The Keynote app on the iPad is a userfriendly application for creating high quality slideshows.

There are three different ways in which the files already created in the computer can be transferred to the iPad. One is to email the file as an attachment and open the email attachment in the iPad using the Keynote app and save it as a Keynote file. The second way is to upload it to the iCloud account and download it directly into the iPad. The third way is to connect the iPad to the computer with the cable and use the iTunes program on the computer to transfer the file to the iPad. One drawback of the Keynote app was its inability to transfer complex equations created in PowerPoint. The "Formula" app, which is a LaTex like application with a user-friendly interface, can be used to re-create the equations for copying and pasting into the Keynote slides.

Since the iPad is connected to the data projector via a VGA adaptor, it is tethered to the podium in the classroom. This is not ideal if one likes to pace during the lecture. The "Keynote Remote" app on the iPhone can be used to remotely advance the slides without being tied to the podium. The Keynoter Remote app can be programmed to show the speaker notes or the next slide depending on the orientation the iPhone is held. There are some limitations for this connections based on the WiFi setup. As long as both iPad and the iPhone are connected to the same WiFi router, the Keynote Remote will work. Unfortunately, with the multitude of wireless routers in the building, it was difficult to establish peerto-peer connection between the iPad and the iPhone. However, if the WiFi is turned-off in both devices, the peer-to-peer connection between the iPhone and the iPad via the Bluetooth will be forced to become active, enabling the remote control of the slideshow. This is not ideal because the access to the internet is now limited to the iPhone's cellular data connection, via tethering, which could become very expensive to use at the prevailing cellular data usage cost.

\subsection{An alternative for the Whiteboard}

The "home button" on the iPad can be double clicked to access recently opened apps. This can be used to switch to another app capable of projecting a drawing app. The "Notes Plus" app is useful for replacing the functions of the whiteboard. The Notes Plus app has a multitude of pen selections with different thicknesses. The background can be plain, ruled, or gridded depending on the need. Anything that is drawn on the page can be retained by opening a new page rather than erasing. There is a link to access the internet and capture parts of a webpage as an image that can be annotated live. This app is an ideal tool for making long derivations that can be saved as .pdf files to be emailed to the students from within the app.

Any .pdf document sent as an email attachment can be opened in Notes Plus app from within the mail app in the iPad. Once opened in Notes Plus, the document can be annotated. This is a useful feature for commenting on data and graphs that already exist in electronic form as a .pdf file.

The Notes Plus app has many automatic features that make it a powerful drawing tool for drawing and inputting text. It has shape recognition capability which enables any hand-drawn lines, boxes, and circles to be converted to perfect geometric shapes. The virtual keyboard of the iPad can also be used to type the text into the page. Any free-hand writing can be converted to text. For those who want to rest their palm on the screen, while writing on the iPad with a stylus, there is a virtual "palm pad" that can be pulled up from the bottom that will prevent the palm from marking the lower part of the screen. The hand-writing is done within a "magnified input box" which automatically advances as more text is written. This enables one to write bigger letters and yet have smaller letters show up on the whiteboard.

The pages created in the Notes Plus app are grouped into a notebook. The entire notebook or selected pages can be emailed to the class. The Notes Plus app also saves to Google docs or Drop Box allowing one to transfer the file to a computer at a later time.

Images captured with the iPad camera can be retrieved into the Notes Plus app for annotating live in the classroom. This is a useful feature for capturing images that exist in a printed form. Images stored in the Photos app in the iPad can also be retrieved into the Notes Plus app for annotating.

Outside the classroom, the Notes Plus app is a powerful tool for documenting group discussions with sketches and text input that can be emailed to the participants, instantly. On field tours, the iPad camera can be used to capture images that can be inserted into the Notes Plus page with annotations to describe the image. 


\subsection{Replacing the Overhead Projector}

The "PaperView" app mimics the traditional overhead projector with the polythene transparency roll. Once a virtual roll is opened in the app, sliding with two fingers up and down will scroll the roll forward and backwards. Pinching the screen in or out with two fingers will zoom in or out without zooming the projected image. This is a useful feature for those who are used to writing in large characters. Any .pdf file sent as an email attachment can be opened in PaperView and inserted as a layer in the virtual roll. The inserted images can be annotated using either a black or a red coloured pen.

Limited pen colours and stroke thicknesses and the inability to delete the virtual rolls created are major limitations of the PaperView app. However, for those who are just migrating from the overhead projector, the PaperView app is simple to use and has a similar look and feel.

\subsection{The iPad as a Document Camera}

The iPad has front and rear cameras. The "Board Cam" app allows one to use either of these cameras to project the image in the field of view live via the data projector. The images appearing on the screen can be annotated and/or labeled in different colours. The projected image is magnified by zooming in the camera electronically from within the Board Cam app. This feature allows one to project small specimens or images.

The Boar Cam app has the ability to capture a Video of the field of view along with the annotations. The annotations can be added while the video capture is paused allowing one to create neatly labeled experimental procedures in the laboratory or the field. The ability to annotate the video during recording is a feature that is not easily accomplished using a camcorder. This feature allows one to record an annotated lab demonstration that can be played prior to an actual lab begins. The ability to project a zoomed in image allows a large number of students to see the demonstration clearly.

In the class room, the Board Cam app can simply be used as a document camera to project a paper document onto a screen. Unlike the document camera, this app allows one to annotate and record the different steps as a video. To capture a good quality image, it is important to ensure that the iPad is placed on a stand or clamped firmly on a holder. Proper lighting of the object is important to get a good projected image.

The Board Cam app can be used as a whiteboard with the ability to draw in multitude of colours and pen thicknesses. However, the written material on the whiteboard can be recorded as video but cannot be saved as a .pdf file.

\subsection{Bringing the lab/field into the classroom}

The Board Cam app has the capability to video stream from any live camera. The "Ustream Broadcaster" app allows one to turn an iPhone/iPad into a webcasting video camera. The Ustream Broadcaster app on the iPhone can be used to stream a lab demonstration live via the Ustream website. The Board Cam app can be used to display the live stream from the Ustream website. The Board Cam app controls can be used to pause the live stream and annotate and continue the stream or advance to the current stream. The Ustream Broadcaster app on the iPhone located in the lab/field can be used to stream live video which can be projected with the Board Cam app to make a powerful teaching tool for demonstrating laboratory/field procedures live in the classroom.

"Facetime" app is another choice for chatting live between the classroom and the field. However, the images cannot be zoomed or annotated and saved when using the Facetime app.

\subsection{Other useful apps for engineering}

The iPad has a growing number of apps that extend the utility of the iPad both in and out of the classroom. The "GraphCalc HD" app allows one to instantly graph functions that can be projected onto the screen. This app has customizable background and graph colours. The graph pane can be scrolled with one finger easily and using a two-finger pinching motion, one can zoom to specific sections of the graph. The "Calculator" app functions in two different ways. In the portrait orientation it is a simple arithmetic calculator. In the landscape mode, the app becomes a powerful scientific calculator.

The "Units" app allows one to do a range of unit conversions, instantly. It also provides the equations used for the unit conversions both in English and SI unit systems. In addition, it provides the etymology of the names of some units.

The "GoodReader" app is a comprehensive annotation tool for marking up .pdf files. Files can be transferred via email, iCloud, or from an FTP site. After editing, it can be remotely synced. It is a great app for marking assignments electronically submitted as a .pdf file. The annotations can be saved with the document to provide feedback to the students.

The "Safari" app is the Apple's version of a browser with the ability to create tabs for different sites. When teaching the design process, the Safari app can be used to access manufacturers' data sheets and specifications to select the appropriate components needed for the design problem. The ability to create different Tabs for different sites prior to the class helps save time that is needed to search for this information during the class. 


\section{STUDENT RESPONSE TO THE iPad USE}

Every course is evaluated anonymously at the end of the term for quality using a Student Evaluation of Educational Quality (SEEQ) form. The instructors have an opportunity to provide supplemental questions in a multiple-choice answer format to get additional feedback from the students. The student survey results are not given to the instructor until the course is completed and the marks are turned in.

To assess the impact of the use of iPad on the students, they were asked to respond to additional questions pertaining to the use of iPad in the classroom as part of their SEEQ evaluation. The responses from 17 students are presented below.

\section{1 iPad as a Whiteboard}

The statement that was put forward was "The iPad was an effective tool as a whiteboard to illustrate additional points.” The student responses are presented in Fig. 1.

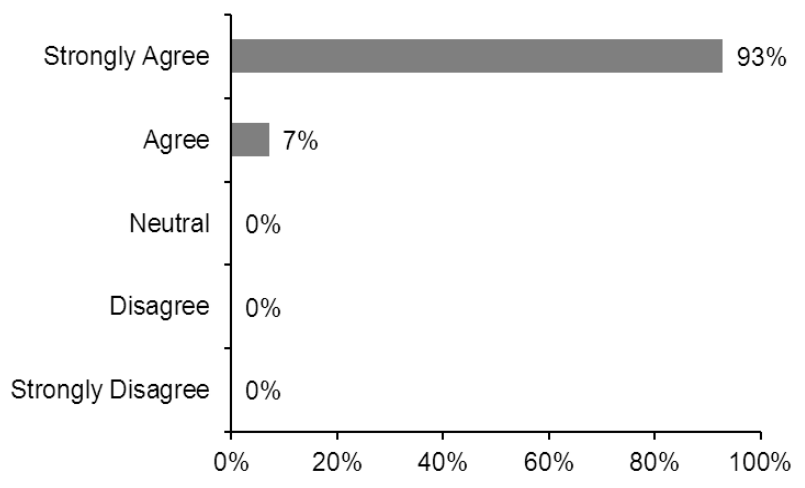

Fig. 1. The iPad to replace the whiteboard

The iPad was used extensively to multitask between the Keynote app to show the slides and the Notes Plus app to elaborate on the points. This required frequent double-clicking of the home button to switch between the apps. The multitasking bar that pops up when the home button is double-clicked contained recently opened apps in the order in which they were used. Sometimes, many different apps were used depending on the content that was under discussion. Clearing the multitask bar prior to each class was found to be important to avoid having to search through a long list of previously used apps. This permitted faster switching between the apps. Students were asked to comment on the ease with which the switching was accomplished in class with the following statement: "The switching between the slides and whiteboard app to illustrate was smooth." Their response is presented in Fig. 2.

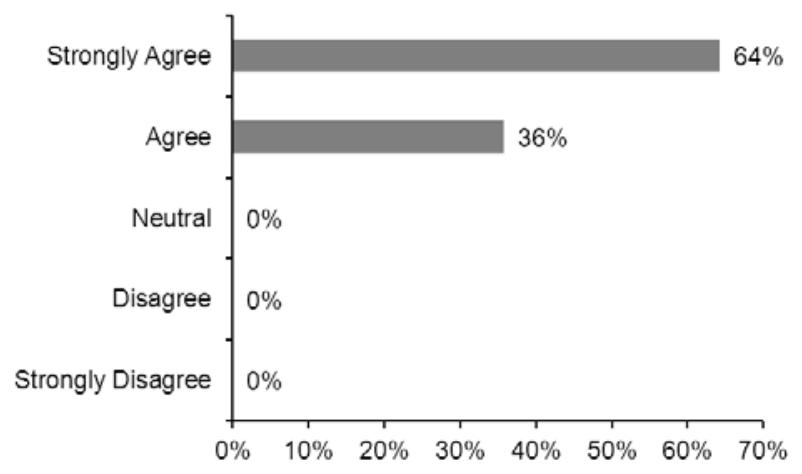

Fig. 2. Ease of multitasking between the apps

\subsection{Navigating the Web with the iPad}

In a class on water management, it was found to be useful to show landscape features in different parts of the world. Showing the Centre Pivot irrigation systems in the Nile Delta transforming the eastern edge of the Sahara desert into lush green circles is key to understanding the need for irrigation. The "Google Earth" app was used to virtually "fly" the students to the Nile Delta so they have an appreciation for the role of irrigation. This app can be used to navigate to different locations to show landscape features and irrigation and drainage systems in different parts of the world. The Google Earth app provides an aerial view of the terrain as if one is flying over the location at different elevations. The iPad responded very smoothly to the navigation (one finger scrolling) and zooming (two-finger pinching) actions. The Safari app was extensively used to show different manufacturers' specification sheets for the components used in the design process. The students were asked to rate the ease of navigation with the following statement: "The iPad was an effective tool for showing Google Earth images and Web content.” Their response is presented as Fig. 2.

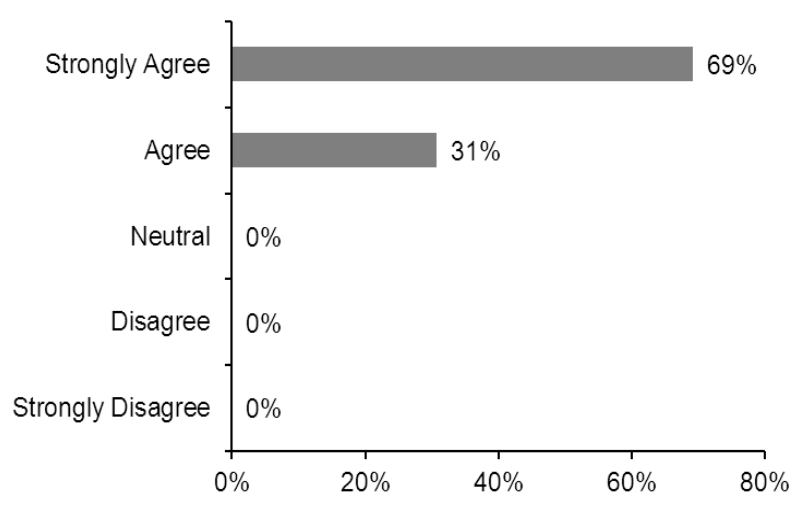

Fig. 3. The ease of navigating the web with the iPad 


\subsection{The iPad as an Overhead Projector}

Sometimes, design problems requiring graphical methods were easily drawn on an overhead transparency. The PaperView app was used to draw on a .pdf graph paper with a stylus. The students were asked to comment on the use of this app with the following statement: "The use of iPad like an overhead projector to illustrate (i.e. the surface ditch design concept) was effective.” Their response is presented in Fig. 4.

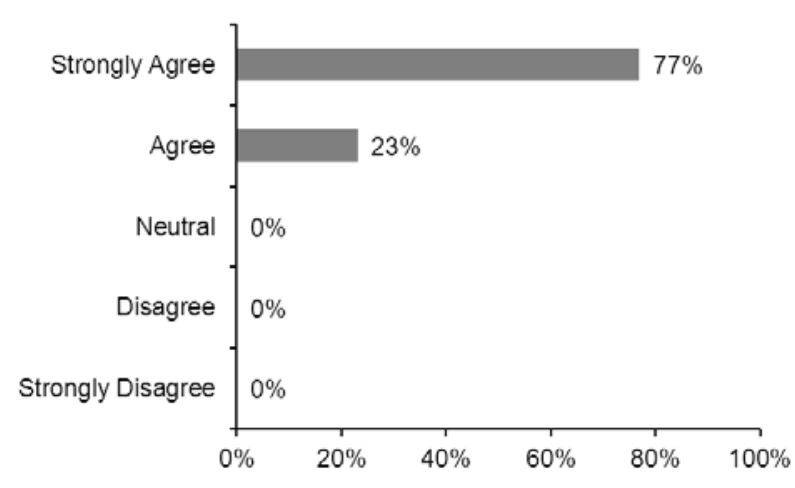

Fig. 4. The iPad to replace an overhead projector

\section{CONCLUSION}

Overall, the use of iPad as an effective technology for delivering course content was a success. The different apps require some practice to use them effectively in a classroom environment. Through live streaming, the iPad also opened-up opportunities to present images and videos from remote locations easily. The student response to the use of the iPad as a teaching aid has been very positive.

Apart from the cost of the iPad, the added cost for the apps was under $C \$ 60$ for a total of about $C \$ 650$ for the basic iPad+WiFi with $16 \mathrm{~Gb}$ storage. The cost of conventional technology which includes an overhead projector, a document camera, a whiteboard, and a computer will cost about C\$ 6500 to equip a single classroom.

\section{Acknowledgements}

I thank the staff of the University Teaching Service for encouraging me to share these ideas with my colleagues. The excellent support provided by the Audio Visual \& Classroom Technology Support personnel over the years is acknowledged.

\section{References}

The following reference and websites present additional information on the use of iPad in teaching:

Sri Ranjan, R. 2012. The evolution to iPad as an effective technology for teaching. Path to Pedagogy 20(3): 26-28.

http://www.ipadineducation.co.uk/iPad_in_Education/We lcome.html

http://wirededucator.wordpress.com/2010/04/15/an-ipadin-every-classroom-teacher-tested-student-approved/

http://www.emergingedtech.com/2011/03/10-excellentipad-applications-for-teachers/

http://www.emergingedtech.com/2012/03/15-favoriteipad-apps-as-selected-by-teachers/

http://teachwithyouripad.wikispaces.com/

http://www.zdnet.com/blog/igeneration/50-resources-foripad-use-in-the-classroom/16126

http://www.whiteboardblog.co.uk/2012/04/anintroductory-guide-to-ipads-for-teachers/

http://www.ipadforums.net/ipad-business-education/ 